Ann. Zootech., I970, 19 (4), 455-459.

NO'TE

\title{
INFLUENCE DE LA TAILLE DU LOT SUR LA QUANTITÉ D'ALIMENT INGÉRE PAR LA BREBIS EN BERGERIE
}

\author{
M. THÉRIEZ et G. MOLENAT \\ Station de Recherches sur l'Élevage des Ruminants, \\ Centre de Recherches de Clermont-Ferrand, 63 -Saint-Genès-Champanelle \\ Institut national de la Recherche agronomique
}

\section{RÉSUMÉ}

On a comparé les quantités de matière sèche ingérée par des brebis en cases individuelles ou en lots de 6 ou de 24 animaux et on a déterminé le nombre minimum de sujets nécessaires pour mesurer ces quantités à 5 p. 100 près.

Sous réserve d'utiliser au minimum I 5 brebis gestantes et 2 I en lactation, les résultats obtenus sont les mêmes que les animaux soient logés en cases individuelles ou en lots.

\section{INTRODUCTION}

Pour établir les plans de rationnement des brebis en bergerie, il est nécessaire de connaître, outre leurs besoins et la valeur alimentaire des aliments offerts, les quantités de matière sèche qu'elles ingèrent suivant Ieur état physiologique.

Or, de telles données sont rares (Forbes et al., I967 ; Forbes, 1969), parce que la majeure partie des travaux de recherche sur l'alimentation du mouton ont été réalisés dans des pays d'élevage extensif (Australie, États-Unis) ou de plein air (Grande-Bretagne).

C'est pourquoi nous avons entrepris d'étudier les variations du niveau d'ingestion de la brebis selon qu'elle est tarie, en gestation ou qu'elle allaite. La période voisine de la mise bas est la plus importante pour la brebis car c'est au cours de celle-ci que les besoins sont les plus difficiles à couvrir.

Avant d'entreprendre l'étude systématique des facteurs modifiant les quantités de matière sèche ingérée, nous nous sornmes proposés, dans l'essai préliminaire rapporté ci-après, de déterminer d'une part le nombre minimum d'animaux nécessaires pour mesurer avec suffisamment de précision la quantité ingérée et, d'autre part le mode de conduite de ces animaux : en case indivi- 
duelle ou en lot. En effet, si pour la vache laitière, les résultats obtenus sur des animaux nourris individuellement peuvent être considérés comme représentatifs de la méthode normale d'élevage, il ne peut pas en être de même pour la brebis, animal qui est toujours élevé en lots.

\section{MATÉRIEL E'T MÉTHODES}

Nous avons comparé les quantités de matière sèche ingérée par 40 brebis dont too étaient logées en cases individuelles, I 2 en 2 lots de 6 et 48 en 2 lots de 24 . Ces brebis, de race Limousine, âgées de 2 à 5 ans, pesaient en moyenne $65 \mathrm{~kg}$ en fin de gestation et $50 \mathrm{~kg}$ après 6 semaines de lactation.

L'agnelage a eu lieu du 8 au 23 mars. L'expérience a duré 98 jours, du début janvier ( $8^{\mathrm{e}}$ semaine avant la mise bas) à la fin avril (6 $6^{\mathrm{e}}$ semaine après la mise bas). Les animaux disposaient chacun d'une place au râtelier et pouvaient tous manger simultanément. Ils étaient logés sous un hangar ouvert sur une face.

Les brebis disposaient à volonté et en permanence d'un foin de pré dont le coefficient de digestibilité de la matière organique était de $5^{8,8} \mathrm{p}$. I oo ce qui correspond à $0,49 \mathrm{UF} / \mathrm{kg}$ de matière séche. A partir de la mise bas, elles ont reçu $200 \mathrm{~g}$ par tête d'un aliment concentré du commerce.

Le foin haché était distribué deux fois par jour et on mesurait les quantités offertes et refusées 5 jours par semaine. Les données de chaque période de 5 jours ont été regroupées et la moyenne obtenue a été considérée comme représentative de la consommation de la brebis au cours de la semaine correspondante, estimation qui est conforme aux observations de BLAXTER et al. (I96I). Nous avons ainsi la consommation des brebis au cours de I 4 périodes successives.

Les quantités offertes étaient ajustées chaque semaine en fonction des refus que l'on maintenait voisins de 15 p. Ioo. Les teneurs en matière sèche du foin, de l'aliment concentré et des refus étaient déterminées deux fois par semaine et les animaux pesés tous les io jours.

\section{RÉSULTATS}

I. Nous avons reporté dans le tableau i les valeurs caractéristiques obtenues sur les 40 brebis en cases individuelles. La quantité de matière sèche de fourrage ingérée par ces animaux diminue en fin de gestation, et n'augmente que très peu après la mise bas.

TABLEAU I

Quantité de matière sèche de fourrage ingérée par des brebis avant et après mise bas

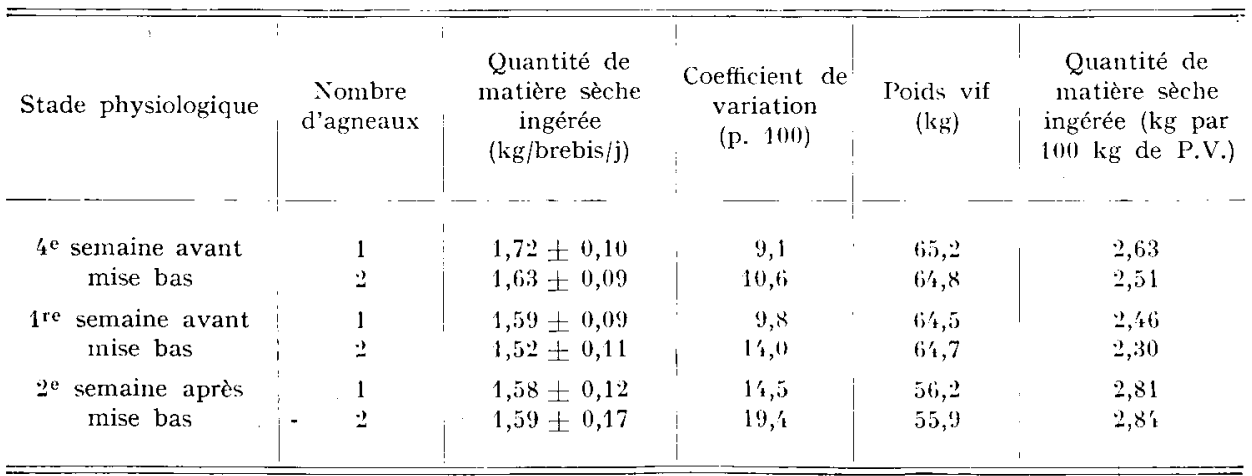


Si les valeurs observées sont rapportées au poids vif (fig. I), la consommation de matière sèche, qui diminue avant l'agnelage, augmente, par contre, fortement après.

Ces variations sont analogues chez les brebis portant un ou deux agneaux, mais sont plus importantes chez les bessonnières. Nous n'avons, par contre, pas observé de différence après la mise bas entre les brebis qui allaitent un ou deux agneaux.

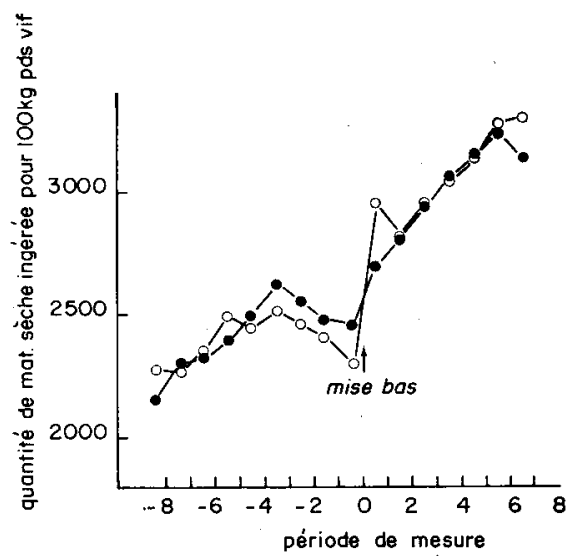

FIG. I. - Quantité de matière sèche ingérée (foin seulèment) pour $100 \mathrm{~kg}$ de poids vif par des brebis en cases individuelles portant puis allaitant 1 ou 2 agneaux

-
0 brebis portant puis allaitant I agneau

L'analyse de variance des résultats obtenus sur ces 4 o brebis, nous a permis de déterminer le nombre minimum d'animaux nécessaires pour estimer, à $5 \mathrm{p}$. roo près, et avec une probabilité d'erreur inférieure à 5 p. Ioo, la quantité de matière sèche ingérée par les brebis. Pour l'ensemble des 14 périodes de mesures, ce nombre minimum s'élève à I 7 pour celles qui portent ou allaitent un agneau et à $\mathbf{~} 8$ pour les bessonnières.

En outre, le comportement individuel des brebis est plus variable après mise bas qu'avant puisqu'il faut $2 \mathrm{I}$ bêtes allaitantes ( $\mathrm{I}$ ou 2 agneaux) pour obtenir la même précision qu'avec I 4 brebis portant I agneau ou I 5 en portant 2.

2. Afin de comparer les quantités de matière sèche ingérée par des brebis en lots à celles que nous avons enregistrées pour des brebis en cases individuelles, nous avons calculé, pour chaque semaine de mesure, la quantité de matière sèche qu'aurait ingérée chaque brebis du lot si elle avait été seule. Nous avons estimé cette quantité d'après le poids vif et le stade physiologique par rapport à la mise bas de chacune des brebis. Nous avons comparé la somme des quantités individuelles à la quantité de matière sèche effectivement consommée par le lot au cours de cette même semaine.

Les animaux en lots consomment des quantités de fourrage qui sont tantôt supérieures, tantôt inférieures aux consommations théoriques obtenues par le calcul (fig. 2). L'écart observé entre consommations théoriques et consommations réelles est significatif dans le cas des lots de 6 animaux ( $\mathrm{P}<\mathrm{o}$, OI pour l'un, $0,01<\mathrm{P}<0,05$ pour l'autre). Il n'est pas significatif dans le cas des lots de 24 brebis. La différence de comportement entre les lots de 6 ou de 24 animaux peut être due au fait qu'un effectif de 6 brebis est trop faible puisqu'il est inférieur au minimum précédemment déterminé alors que nous nous rapprochons de ce minimum avec les lots de 24 bêtes 


\section{CONCLUSIONS}

Les résultats des mesures des quantités de matière sèche ingérée par des brebis en cases individuelles, sous réserve d'utiliser le nombre minimum d'animaux nécessaires, peuvent être généralisés à la pratique normale de l'élevage en lots.

Réciproquement, nous pourrons réunir des brebis en lot pour mesurer, avec la même précision, les quantités de matière sèche qu'elles auraient consommé en moyenne si elles avaient été alimentées individuellement.

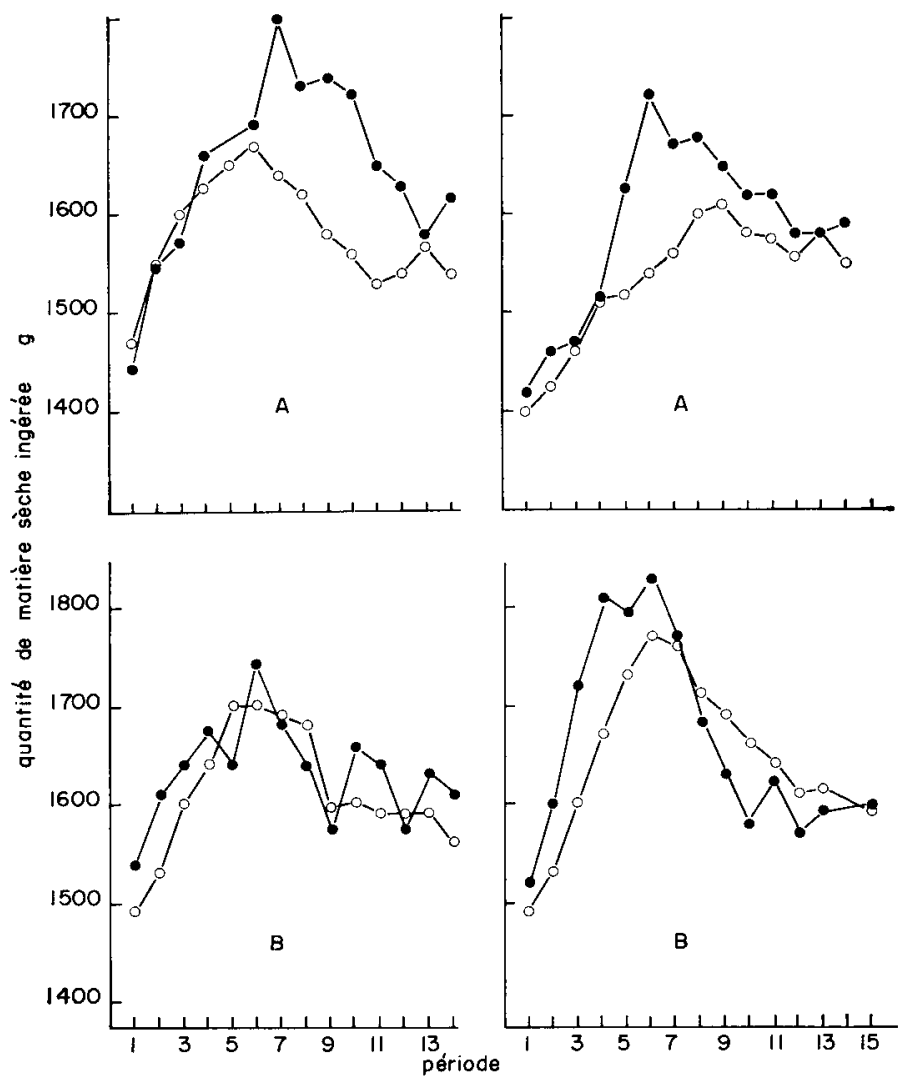

FIG. 2. - Comparaison de la quantité théorique de matière sèche ingérée et de la consommation réelle par des brebis en lot de 6 (courbes A) ou en lots de 24 (courbes B)

- - - consommation réelle

○ _._. o consommation théorique

Reçu pour publication en septembre 1970. 


\section{SUMMARY}

INFLUENCE OF THE GROUP SIZE UPON THE AMOUNT
OF FOOD INGESTED BY EWES KEPT IN SHEEP BARNS

The amounts of dry matter ingested by the ewes kept in individual pens or in groups of 6 or 24 animals were compared and the minimun number of animals necessary to measure these amounts was determined with an accuracy of 5 p. 100.

Provided a minimum number of 15 pregnant ewes and 21 lactating ewes, the results obtained were the same whether the animals were kept in individual pens or in groups.

\section{RÉFÉRENCES BIBLIOGRAPHIQUES}

Blaxter K. L., Wainman F. W., Wilson R.S., I96I. The regulation of food intake by sheep. Anim. Prod., 3, 5I-62.

Forbes J. M., Rees J. K. S., Boaz T. G., 1967. Silage as a feed for pregnant ewe. Anim. Prod., 9, 399-408.

Forbes J. M., I969. A note of the voluntary feed intake of lactating ewes, their milk yield and the grow th rate of their lambs. Anim. Prod., 11, 263-266. 